\title{
A Feasibility Test on Preventing PRMDs Based on Deep Learning
}

\author{
So-Hyun Park, ${ }^{1}$ Sun-Young Ihm, ${ }^{1}$ Aziz Nasridinov, ${ }^{2}$ Young-Ho Park ${ }^{1, *}$ \\ ${ }^{1}$ Dept. of IT Engineering, Sookmyung Women's University, Seoul, 04310, South Korea, +82-10-3907-9274 \\ \{shpark, sunnyihm, yhpark\}@ sookmyung.ac.kr \\ ${ }^{2}$ Dept. of Computer Science, Chungbuk National University, Cheong-Ju, 28644, South Korea \\ aziz@ chungbuk.ac.kr \\ ${ }^{*}$ Corresponding Author
}

\begin{abstract}
This study proposes a method to reduce the playing-related musculoskeletal disorders (PRMDs) that often occur among pianists. Specifically, we propose a feasibility test that evaluates several state-of-the-art deep learning algorithms to prevent injuries of pianist. For this, we propose (1) a C3P dataset including various piano playing postures and show (2) the application of four learning algorithms, which demonstrated their superiority in video classification, to the proposed $\mathrm{C} 3 \mathrm{P}$ datasets. To our knowledge, this is the first study that attempted to apply the deep learning paradigm to reduce the PRMDs in pianist. The experimental results demonstrated that the classification accuracy is $80 \%$ on average, indicating that the proposed hypothesis about the effectiveness of the deep learning algorithms to prevent injuries of pianist is true.
\end{abstract}

\section{Introduction}

Playing-related musculoskeletal disorders (PRMDs) are a frequent occurrence among musical instrument players; such disorders can profoundly affect the technique of piano players (Dommerholt 2010). The medical community and piano player association have long noted that utilizing a wrong or a stiff posture can be a major cause of PRMDs (Zaza 1998). To prevent PRMDs, a variety of methods have been proposed including the analysis of players' posture through wearable and physical sensors. However, the dataset used in these studies (Winges and Furuya 2015), (Park et al. 2016) do not consider various situations of the pianist, and are thus biased in posture classification.

This paper proposes the following contributions. (1) A classical piano performance posture (C3P) dataset, which unlike existing datasets contains various data, such as diverse environments, pianists, songs, and information of major/minor students. (2) We applied the state-of-the-art deep learning algorithms on video classification to the $\mathrm{C} 3 \mathrm{P}$ dataset and performed a feasibility test that recommends a suitable algorithm to determine the correct posture for a pianist.

Copyright (C) 2019, Association for the Advancement of Artificial Intelligence (www.aaai.org). All rights reserved.

\section{Our Approach}

In this section, we first describe the details of the proposed $\mathrm{C} 3 \mathrm{P}$ dataset, and then explain posture classification.

\section{C3P Dataset}

The total number of clips is 102 and the mean clip length is $7.5 \mathrm{~s}$. The numbers of pianist and music pieces are 15 each. The clips in one part have the same features, such as the pianist. The videos are obtained via YouTube, where the clips contain a fixed frame rate of 25 frame per second and resolution of $720 \times 576$, respectively. The videos are stored as .avi files processed using DV PAL codec existing in Adobe Premiere Pro. We intentionally removed the audio. The dataset contains web videos obtained from various environments that include camera motion, various lighting conditions, partial occlusion, low-quality frames, etc. Table 1 shows the summary of proposed C3P dataset.

\begin{tabular}{|c|c|}
\hline Number of players & 15 \\
\hline Mean clip length & $7.5 \mathrm{sec}$ \\
\hline Clips & 102 \\
\hline Min clip length & $5 \mathrm{sec}$ \\
\hline Max clip length & $12 \mathrm{sec}$ \\
\hline Number of music piece & 17 \\
\hline Frame rate & $25 \mathrm{fps}$ \\
\hline Resolution & $720 \times 576$ \\
\hline Total duration & $766 \mathrm{sec}$ \\
\hline Audio & no \\
\hline
\end{tabular}

Table 1: Summary of characteristics of C3P

The purpose of this study is to classify the postures of pianists into two kinds, correct and incorrect, as shown in Figure 1, presenting sample frames of two action classes from C3P. The injury-related poses were classified with the help of a specialist who had more than 10 years of experience in piano education field.

\section{Video Classification}

Figure 2 shows the posture classification procedure. We used four deep learning algorithms that stand out in the field of video classification. That is, we used 2D Convolutional Networks $(2 \mathrm{D} \mathrm{CNN})$ that classifies the image frame by frame, 

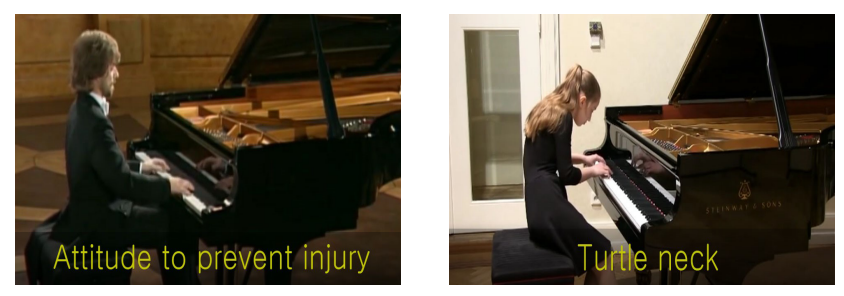

Figure 1: Sample frames for PRMDs posture classes of C3P

and uses Inception V3 pretrained with the ImageNet dataset. Next, we used a transfer learning procedure to retrain the inception according to the proposed $\mathrm{C} 3 \mathrm{P}$ dataset. Further, we used Convolutional 3D (C3D) (Tran et al. 2015) and longterm recurrent convolution network (LRCN) models that utilize temporal features as well as spatial features of videos. In the case of the LRCN model, the spatial feature extracted from the $2 \mathrm{D} C \mathrm{CNN}$ is converted into a sequence form, which is then passed to the long short-term memory. Lastly, we used the multi-layer perceptron (MLP) model, which shows excellent performance for classifying two classes, in combination with $2 \mathrm{D}$ CNN. This model, similar to the LRCN model, converts features extracted from $2 \mathrm{D}$ CNN into a sequence form and passes them to the MLP.

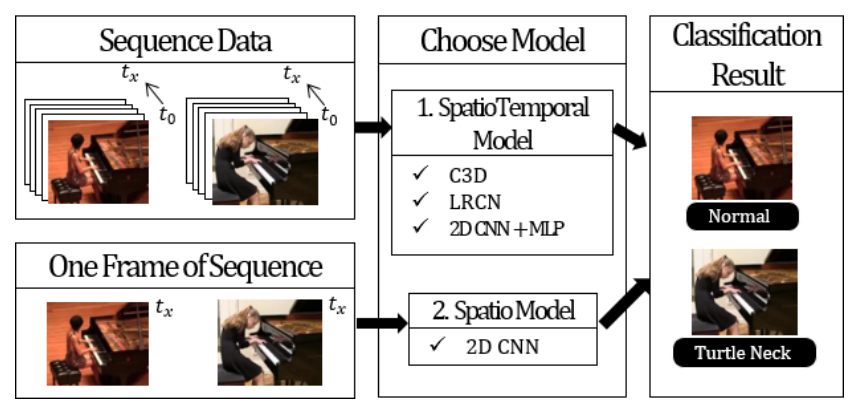

Figure 2: PRMDs posture classification procedure

\section{Experiment Results}

We conducted experiments that applied the four aforementioned deep learning algorithms on the $\mathrm{C} 3 \mathrm{P}$ dataset and compared the classification accuracy. The C3P dataset was divided into the validation and training datasets, which were set at $33 \%$ and $67 \%$, respectively. In addition, we divided the dataset for performing $\mathrm{k}$-fold validation. The accuracy range was between 0 and 1 . Table 1 demonstrates the results of the experiments on the accuracy of injury-related posture classification by using various deep learning models. Moreover, the table shows the result of the feasibility test. For experiments, we executed 3-fold validation. According to the experimental results, the 2D CNN and 2D CNN + MLP models show competitive accuracy results of 0.8254 and 0.8771 , respectively.

\begin{tabular}{|c|c|c|c|c|}
\hline Model & Iter. 1 & Iter. 2 & Iter. 3 & Average \\
\hline 2D CNN & 0.99 & 0.7544 & 0.7319 & 0.8254 \\
\hline C3D & 0.7031 & 0.7424 & 0.7222 & 0.7225 \\
\hline LRCN & 0.66 & 0.7424 & 0.75 & 0.7174 \\
\hline 2D CNN + MLP & 0.875 & 0.9091 & 0.8472 & 0.8771 \\
\hline
\end{tabular}

Table 2: The result of PRMDs posture classification accuracy

\section{Conclusion}

This study verified the feasibility of a piano-playing injury prevention system using deep learning. For effective analysis, a C3P dataset, including diverse environments, was proposed; it solves the problems of models presented in previous related studies, in which the type of data was limited. We also applied state-of-the-art video classification deep learning models to the $\mathrm{C} 3 \mathrm{P}$ dataset. The experimental results show an average classification accuracy of $80 \%$, which indicates that the proposed hypothesis about the effective use of deep learning algorithms to prevent PRMDs is acceptable. The proposed method is the first in our knowledge that uses deep learning to prevent injuries to pianists playing a piano, and this is the first study that systematically uses deep learning in the field of art. Future studies will be conducted to improve the accuracy of additional data acquisition and deep learning models.

\section{Acknowledgement}

This research was supported by Basic Science Research Program through the National Research Foundation of Korea (NRF) funded by the Ministry of Education, Science and Technology (NRF-2018R1D1A1B07046550). In addition, this work was supported by Institute for Information \& communications Technology Promotion (IITP) grant funded by the Korea government (MSIP) (No.2016-0-00406, SIAT CCTV Cloud Platform).

\section{References}

Dommerholt, J. 2010. Performing arts medicineinstrumentalist musicians, part ii-examination. Journal of bodywork and movement therapies 14(1):65-72.

Park, S.-H.; Hong, G.-S.; Park, S.-W.; Nasridinov, A.; Park, I.-J.; Kim, B.-K.; and Park, Y.-H. 2016. A feasibility study of ballet education using measurement and analysis on partial features of still scenes. International Journal of Distributed Sensor Networks 12(12):1550147716681794.

Tran, D.; Bourdev, L.; Fergus, R.; Torresani, L.; and Paluri, M. 2015. Learning spatiotemporal features with 3d convolutional networks. In Proceedings of the IEEE international conference on computer vision, 4489-4497.

Winges, S. A., and Furuya, S. 2015. Distinct digit kinematics by professional and amateur pianists. Neuroscience 284:643-652.

Zaza, C. 1998. Playing-related musculoskeletal disorders in musicians: a systematic review of incidence and prevalence. Canadian medical association journal 158(8):1019-1025. 